\title{
Balo's concentric sclerosis: a clinical case study of brain MRI, biopsy, and proton magnetic resonance spectroscopic findings
}

\author{
Mee Ohk Kim, Sang Ahm Lee, Choong Gon Choi, Joo Ryoung Huh, \\ Myoung Chong Lee
}

\begin{abstract}
The antemortem diagnosis of Balo's concentric sclerosis was made in a 52 year old woman with subacute right hemiparesis on the basis of brain MRI and stereotactic brain biopsy, which showed multiple ring-like lesions of lamellated demyelination alternating with spared white matter. Proton magnetic resonance spectroscopy (1H-MRS) was carried out one and nine months after the onset of illness. The first ${ }^{1}$ H-MRS showed a decreased $\mathbf{N}$-acetyl aspartate peak, an increased choline peak, presence of large lipid peaks, and high resonance at $1.4 \mathrm{ppm}$. The second ${ }^{1}$ H-MRS disclosed changes such as a decrease of lipid signal, a decrease of resonance at $1.4 \mathrm{ppm}$, and an increase in the myoinositol peak. These findings are similar to those reported for multiple sclerosis. It seems that this is the first report of ${ }^{1} H-M R S$ findings in Balo's concentric sclerosis.
\end{abstract}

$(\mathcal{F}$ Neurol Neurosurg Psychiatry 1997;62:655-658)

Keywords: Balo's concentric sclerosis; brain MRI; brain biopsy; proton magnetic resonance spectroscopy

Department of

Neurology

M O Kim

$S$ A Lee

M C Lee

Department of

Diagnostic Radiology

C G Choi

Department of

Diagnostic Pathology,

Asan Medical Center,

Song-Pa PO Box 145, Seoul, 134-600, South Korea

J R Huh

Correspondence to:

Dr S A Lee, Department of Neurology, Asan Medical Center, College of Medicine, University of Ulsan, Song-Pa PO Box 145, Seoul, 134-600, South Korea.
Balo's concentric sclerosis is a rare demyelinating disorder. It is pathologically characterised by alternating bands of demyelinated and myelinated white matter. ${ }^{1-3}$ The pathogenesis of these banded patterns are still poorly understood. Proton magnetic resonance spectroscopy ( $\left.{ }^{1} \mathrm{H}-\mathrm{MRS}\right)$ offers a noninvasive way to study biochemical changes that may help to understand the pathogenesis.

Recently, some patients with Balo's concentric sclerosis have been diagnosed at antemortem examination using brain MRI and biopsy. ${ }^{4-9}$ However, in vivo ${ }^{1} \mathrm{H}-\mathrm{MRS}$ findings in Balo's concentric sclerosis has not yet been described.

We present ${ }^{1} \mathrm{H}$-MRS findings in a patient with Balo's concentric sclerosis, diagnosed by MRI and stereotactic brain biopsy.

\section{Case report}

A 52 year old woman presented with subacute right hemiparesis. She was well until two weeks before admission, when she noted right leg weakness. By the next week she had developed weakness of her right arm.

Neurological examination disclosed a right hemiparesis with right facial weakness of a central type. The deep tendon reflexes were increased with extensor plantar response on the right side. A snout reflex was found.

Analysis of CSF showed mild pleocytosis (9 white blood cells $/ \mathrm{mm}^{3}$ ), normal total protein, and no oligoclonal band. The IgG index in CSF was 0.79. Brainstem auditory evoked potentials, pattern shift visual evoked potentials, and somatosensory evoked potentials were all normal. Routine haematological and biochemical tests were normal.

Brain MRI showed multiple lamellated white matter lesions with marginal gadolinium enhancement in the right frontal lobe and bilateral centrum semiovale (fig 1A). Treatment was started with intravenous dexamethasone. The right hemiparesis began to improve during the first week of treatment. Brain MRI was repeated 14 days after treatment. The ring-like pattern of lesions was not changed but the right frontal lesion was considerably increased in size despite clinical improvement. The brain biopsy was done on the 22nd day in hospital. The right hemiparesis gradually resolved over the next five months. She has done well without relapse for more than 17 months.

\section{Pathological findings}

An MR guided stereotactic needle biopsy was obtained from the left centrum semiovale lesion. Haematoxylin-eosin and luxol fast blue staining of biopsied brain sections showed the alternation of bright and dark bands under light microscopy ( $\times 40)$; the dark bands represented preserved myelinated areas and the bright bands demyelinated ones. An immunohistochemical study with HAM 56 antibody disclosed much increased numbers of macro- 
Figure 1 (A) The abnormal signal intensities on proton weighted imaging were composed of repetitive alternations of inner higher signal intensities and surrounding outer lower signal intensities which looked like lamellated rings. (B) Immunohistochemical study using HAM 56 antibody for macrophage disclosed increased numbers of macrophages only in the demyelinated areas (D) indicative of repetitive alternations of myelinated (M) and demyelinated areas (D).

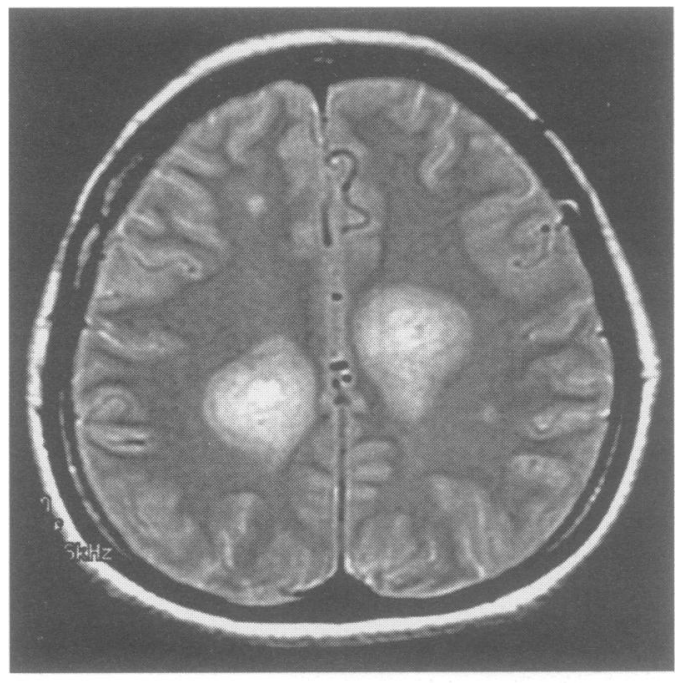

A

phages, but only in the demyelinated areas (fig 1B). In the demyelinated areas, reactive astrocytosis, foamy macrophages, and perivenular mononuclear cells infiltration were noted under light microscopy $(\times 200)$.

Proton magnetic resonance spectroscopy ('H-MRS)

In vivo ${ }^{1} \mathrm{H}-\mathrm{MRS}$ using a GE $1.5 \mathrm{~T}$ Signa $\mathrm{MR}$ system was performed one and nine months after the onset of illness. Local proton spectra were recorded with stimulated echo acquisition mode (STEAM) sequences (TR $3.0 \mathrm{~s}$, TE $30 \mathrm{~ms}$, 64 AVG, volume 8-9 ml) before gadolinium enhancement.

The first ${ }^{1} \mathrm{H}-\mathrm{MRS}$ was obtained from the left enhancing centrum semiovale lesion. It showed a decrease in $\mathrm{N}$-acetyl aspartate:creatine ratio and an increase in choline:creatine ratio. The more striking findings were the presence of large resonances at $0 \cdot 9,1 \cdot 3$, and $1.4 \mathrm{ppm}$ (fig 2A).

The second ${ }^{1} \mathrm{H}-\mathrm{MRS}$ was obtained from the right non-enhancing centrum semiovale lesion because brain biopsy was done at the left side. Compared with the first ${ }^{1} \mathrm{H}-\mathrm{MRS}$, the large resonances at $0.9,1.3$, and $1.4 \mathrm{ppm}$ were very reduced and the myoinositol peak was significantly increased (fig 2B). There were no significant changes in $\mathrm{N}$-acetyl aspartate and choline peaks.

\section{Discussion}

The antemortem diagnosis of Balo's concentric sclerosis was made in our patient on the basis of brain MRI and stereotactic biopsy, which showed multiple ring-like lesions of lamellated demyelination alternating with spared white matter. These findings are the hallmarks of Balo's concentric sclerosis. The pathogenesis of these banded patterns in large cerebral lesions are still poorly understood. Recently, Yao et al considered that oligodendroglial loss is important in the pathogenesis of demyelination and that partially myelinated areas probably represent stages of ongoing myelin breakdown rather than remyelination

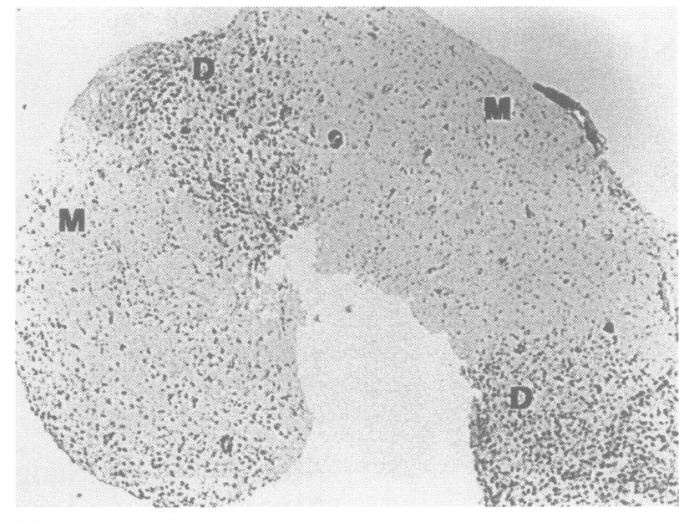

B

of previously demyelinated areas. ${ }^{10}$

${ }^{1} \mathrm{H}-\mathrm{MRS}$ may provide new insights into various brain diseases, especially into demyelinating diseases. There are several reports of the value of ${ }^{1} \mathrm{H}-\mathrm{MRS}$ in diagnosing multiple sclerosis, monitoring its progression, and evaluating its response to treatment. ${ }^{11-18}$ Balo's concentric sclerosis has apparently not yet been studied with ${ }^{1} \mathrm{H}-\mathrm{MRS}$.

In our case ${ }^{1} \mathrm{H}$-MRS was initially studied at the acute enhancing lesion one month after the onset of symptom. The characteristic findings were a decrease in the $\mathrm{N}$-acetyl aspartate:creatine ratio, an increase in choline: creatine ratio, and a presence of high resonances at $0.9,1.3$, and $1.4 \mathrm{ppm}$. These spectroscopic findings are very similar to those found in acute plaques of multiple sclerosis. ${ }^{11-1315} \mathrm{~N}$-acetyl aspartate has been implicated as a neuronal marker and its decrease may be explained by neuronal or axonal loss and gliosis. ${ }^{11-15}$ The increase in choline may reflect the breakdown of myelin membrane phospholipids. ${ }^{11-1315}$ The short echo ${ }^{1} \mathrm{H}-\mathrm{MRS}$ can detect myelin breakdown products, which probably cause an increase in lipid resonances. Short echo spectra from acute enhancing multiple sclerosis plaques invariably show the presence of large lipid resonances at 0.9 and $1.3 \mathrm{ppm}$. These resonances are not due to extra voxel contamination from the scalp ${ }^{13}$ and have been assigned to the methyl and methylene groups of lipid. ${ }^{19} \mathrm{~A}$ resonance at $1.4 \mathrm{ppm}$ was very prominant in our case. It is not yet clear which metabolite produces this resonance. There is some evidence that the polypeptide thymosin 4 is found in macrophages and in a subset of oligodendrocytes, and produces resonances in this region of the spectrum. ${ }^{20}$ Davie et al showed that this resonance has a longer T2 than the methylene group at $1.3 \mathrm{ppm}$ because it was more prominant at TEs of $20-30 \mathrm{~ms}^{13}$

Follow up ${ }^{1} \mathrm{H}-\mathrm{MRS}$ showed changes in spectra from the previous one. The relevant differences were a remarkable decrease of lipid signal, the resonance at $1.4 \mathrm{ppm}$, and the increase in the myoinositol peak. There were no significant changes in $\mathrm{N}$-acetyl aspartate and choline peaks. Some reports suggest that the time course of increased lipid resonance is compatible with the histologically determined time course of disappearance of lipid laden 
Figure 2 (A) This first
$1 H-M R S(T R=3 \cdot 0 \mathrm{~s}$ $1 H-M R S(T R=3.0 \mathrm{~s}$, lipid signals at 0.9 and $1 \cdot 3$ ppm, a large resonance at $1.4 \mathrm{ppm}$ (white arrow), a decrease in $N A A$ :creatine ratio, and an increase in choline:creatine ratio. (B) The second ${ }^{1} H$-MRS (TR $=3.0 \mathrm{~s}, T E=30 \mathrm{~ms}$ ), nine months after the initial symptoms shows changes such as a decrease in lipid signal, a decrease in the resonance at 1.4 ppm, and an increase in myoinositol peak. There are no significant changes in NAA and choline peaks. $N A A=N$-acetyl aspartate; $m l=$

myoinositol; Cho $=$ choline; $\mathrm{Cr}=$ creatine.

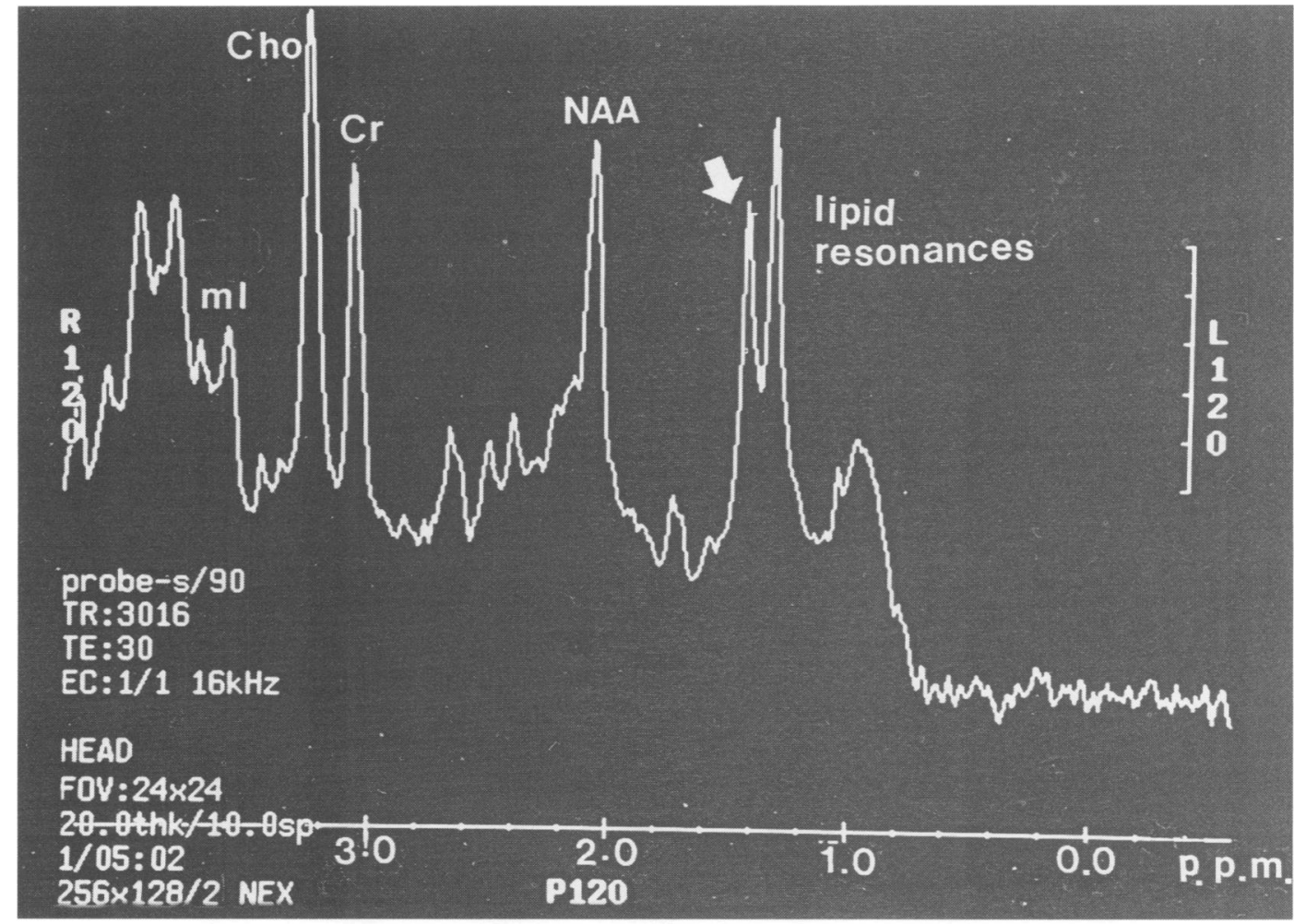

A

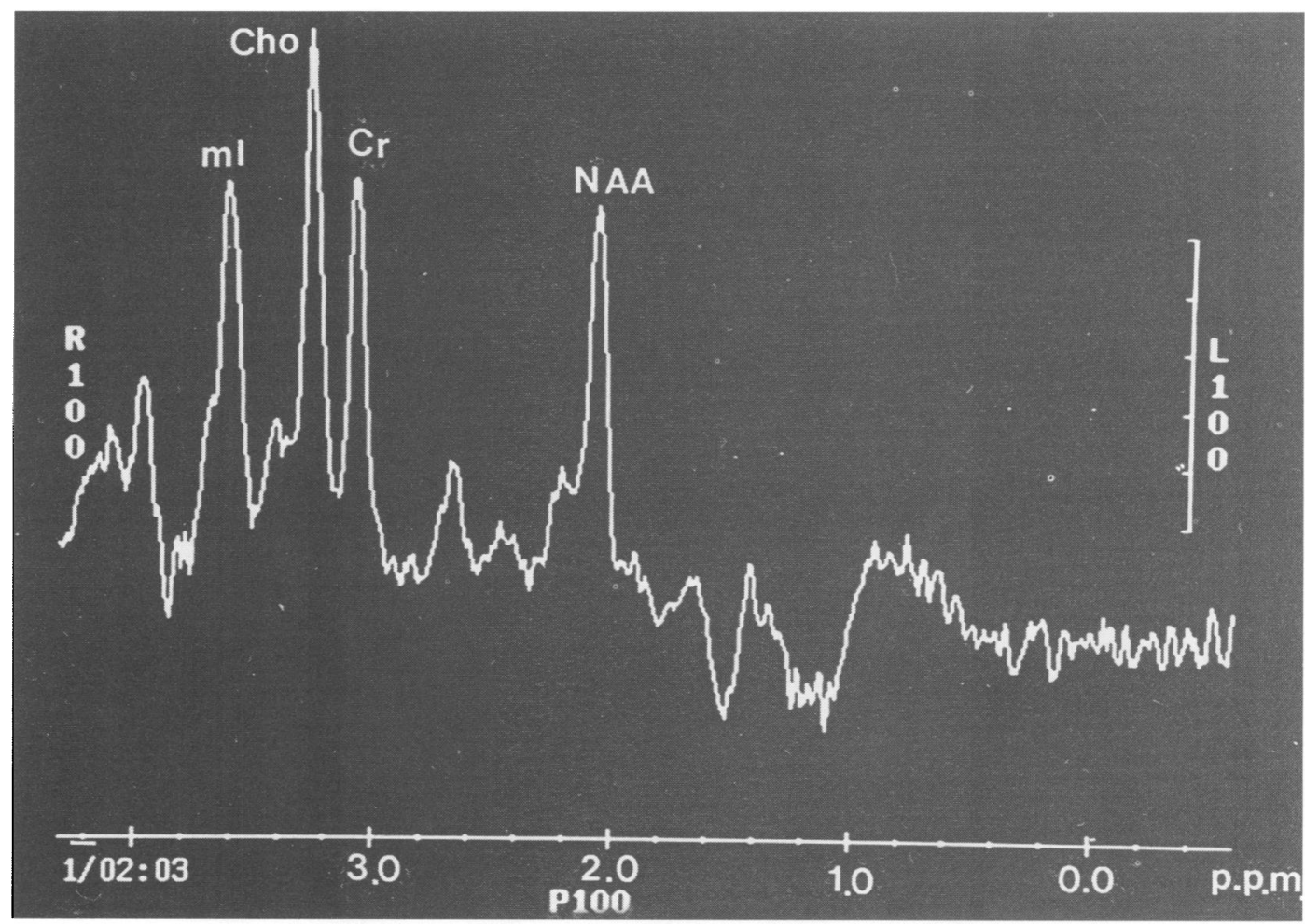

B

macrophages from areas of acute myelin destruction. ${ }^{13}$ Myoinositol has been reported to be present only in glial cells and it was suggested that it may be used as a glial marker. ${ }^{18}$ This increase of myoinositol was attributed to the process of gliosis, suggesting the healing phase of demyelinating plaque. ${ }^{17}{ }^{18}$ Increase of myoinositol in vivo have been seen in chronic as well as acute lesions. ${ }^{1317} 18$

These characteristic ${ }^{1} \mathrm{H}-\mathrm{MRS}$ findings in our case, which are similar to those reported in multiple sclerosis, may be useful in understanding the pathogenesis of Balo's concentric sclerosis. Also, ${ }^{1} \mathrm{H}$-MRS may have additional diagnostic value in allowing brain biopsy to be avoided in the diagnosis of Balo's concentric sclerosis if ${ }^{1} \mathrm{H}-\mathrm{MRS}$ is combined with typical MRI findings. Neurology and Psychiatry 1928;19:242-64.

2 Kuroiwa Y. Concentric sclerosis. In Vinken PJ, Bruyn GW, Klawans HL, eds. Handbook of clinical neurology. Amsterdam: North-Holland, 1985;47:409-17.
1 Balo J. Encephalitis periaxialis concentrica. Archives of 
3 Itoyama Y, Tateishi J, Kuroiwa Y. Atypical multiple sclerosis with concentric or lamellar demyelinated lesions: two Japanese patients studied post mortem. Ann Neurol 1985, 17:481-7.

4 Garbern J, Spence AM, Alvord Jr EC. Balo's concentric demyelination diagnosed premortem. Neurology 1986;36: $1610-4$

5 Spiegel M, Kruger H, Hofmann E, Kappos L. MRI study of Balo's concentric sclerosis before and after immunoof Balo's concentric sclerosis before and after

6 Nandini M, Devi MG, Shankar SK, Mustare VB, Ravi V. Balo's concentric sclerosis diagnosed intravitam on brain Balo's concentric sclerosis diagnosed intravita

7 Revel MP, Valiente E, Gray F, Beges C, Degos JD, Brugieres $P$, Gaston A. Concentric MR patterns in multiple sclerosis. $\mathcal{F}$ Neuroradiol 1993;20:252-7.

8 Korte JH, Bom EP, Vos LD, Breuer TJM, Wondergem JHM. Balo's concentric sclerosis: MR diagnosis. AfNR Am $\mathcal{F}$ Neuroradiol 1994;15:1284-5.

9 Gharagozloo AM, Poe LB, Collins GH. Antemortem diagnosis of Balo's concentric sclerosis: correlative MR imaging and pathologic features. Radiology 1994:191:817-9.

10 Yao DL, Webster HD, Hudson LD, Brenner M, Liu DS, Escobar AI, Komoly S. Concentric sclerosis (Balo): Morphometric and in situ hybridization study of lesions in six patients. Ann Neurol 1994;35:18-30.

11 Richards TL. Proton MR spectroscopy in multiple sclerosis: value in establishing diagnosis, monitoring progression, and evaluating therapy. AfR Am $\mathcal{f}$ Roentgenol 1991; sion, and eval

12 Davie CA, Hawkins CP, Barker GJ, Brennan A, Tofts PS Miller DH, McDoland WI. Detection of myelin break- down products by proton magnetic resonance spectroscopy [letter]. Lancet 1993;341:630-1.

13 Davie CA, Hawkins CP, Barker GJ, Brennan A, Tofts PS, Miller DH, McDonald WI. Serial proton magnetic resonance spectroscopy in acute multiple sclerosis lesions. Brain 1994;117:49-58.

14 Davies SEC, Newcombe J, Williams SR, McDonald WI, Clark JB. High resolution proton MR spectroscopy of multiple sclerosis lesions. $\mathcal{F}$ Neurochem 1995;64:742-8.

15 Roser W, Hagberg G, Mader I, Brunnschweiler H, Radue EW, Seelig J, Kappos L. Proton MRS of gadoliniumenhancing MS plaques and metabolic changes in normal enhancing MS plaques and metabolic changes in normal

appearing white matter. Magn Reson Med 1995;33:81 1-7.
16 Pan JW, Hetherington HP, Mason GF, Whitaker JN, Poshost GM. Evaluation of multiple sclerosis by high field spectroscopic imaging. Proceedings of the Society of Magnetic Resonance in Medicine 1993;12:1552.

17 Zhu G, Allen PS, Koopmans R, Li DK, Patty DW. A marked elevation of inositol in MS lesions. Proceedings of the Society of Magnetic Resonance in Medicine 1992;11:1948.

18 Brand A, Leibfritz D. Metabolic markers in glial cells for differentiation of brain tissues. Proceedings of the Society of Magnetic Resonance in Medicine 1992;11:649.

19 Callies R, Sri-pathmanathan RM, Ferguson DYP, Brindle $K M$. The appearance of neutral lipid signals in the $1 \mathrm{H}$ NMR spectra of a myeloma cell line correlates with the induced formation of cytoplasmic lipid droplets. Magn induced formation of cytop
Reson Med 1993;29:546-52

20 Kauppinen RA, Nissinen T, Karkkainen A-M, Pirttila TRM, Palvimo J, Kokko $H$. Detection of thymosin 4 in
TRM situ in a guinea pig cerebral cortex preparation using $1 \mathrm{H}$ NMR spectroscopy. F Biol Chem 1992;267:9905-10. 